\title{
Gram's Law and the Argument of the Riemann Zeta Function ${ }^{1)}$
}

\author{
M.A. Korolev \\ Steklov Mathematical Institute \\ Gubkina str., 8, \\ 119991, Moscow, Russia \\ hardy_ramanujan@mail.ru, korolevma@mi.ras.ru
}

\begin{abstract}
Annotation. Some new statements concerning the behavior of the argument of Riemann zeta function at the Gram points are proved. We apply these statements to the proof of Selberg's formulas connected with Gram's Law.

Keywords: Gram's law, Gram's rule, Gram points, argument of the Riemann zeta function, Selberg's formulas.
\end{abstract}

\section{$\S 1$. Introduction}

Since the notion 'Gram's Law' has different sense in different papers, it seems reasonable to begin this paper with short survey. This survey contains basic results concerning the peculiar phenomenon observed by Jörgen Pedersen Gram [1] in 1903.

For the following, we need several definitions. Suppose $t>0$ and let $\vartheta(t)$ be an increment of any fixed continuous branch of the argument of the function $\pi^{-s / 2} \Gamma\left(\frac{s}{2}\right)$ as $t$ varies along the segment connecting the points $s=\frac{1}{2}$ and $s=\frac{1}{2}+i t$. Then Hardy's function $Z(\tau)=e^{i \vartheta(\tau)} \zeta\left(\frac{1}{2}+i \tau\right)$ is real for real $\tau$ and it's real zeros coincide with the ordinates of zeros of $\zeta(s)$ lying on the critical line Re $s=\frac{1}{2}$. Further, if $t$ is not an ordinate of a zero of $\zeta(s)$ then the function $S(t)=\pi^{-1} \arg \zeta\left(\frac{1}{2}+i t\right)$ is defined as an increment of any continuous branch of $\pi^{-1} \arg \zeta(s)$ along the polygonal arc connecting the points $2,2+i t$ and $\frac{1}{2}+i t$. In the opposite case, $S(t)$ is defined by the relation

$$
S(t)=\frac{1}{2} \lim _{h \rightarrow 0}(S(t+h)+S(t-h)) .
$$

Let $N(t)$ be a number of zeros of $\zeta(s)$ in the rectangle $0<\operatorname{Im} s \leqslant t, 0 \leqslant \operatorname{Re} s \leqslant 1$ counted with multiplicities. At the points of discontinuity $N(t)$ is defined as follows:

$$
N(t)=\frac{1}{2} \lim _{h \rightarrow 0}(N(t+h)+N(t-h))
$$

The equation

$$
N(t)=\frac{1}{\pi} \vartheta(t)+1+S(t)
$$

holds true for any $t>0$ and is called as Riemann - von Mangoldt formula.

By $\varrho_{n}=\beta_{n}+i \gamma_{n}$ we denote complex zeros of $\zeta(s)$ lying in the upper half-plane and ordered as follows: $0<\gamma_{1}<\gamma_{2}<\ldots \leqslant \gamma_{n} \leqslant \gamma_{n+1} \leqslant \ldots$. Finally, let $c_{n}$ be a positive zeros of $Z(t)$ numbered in ascending order and counted with their multiplicities.

\footnotetext{
1) This research was supported by the Russian Fund of Fundamental Research (grant no. 11-01-00759a).
} 
Though first three positive ordinates of zeros of $\zeta(s)$ had been counted already by Riemann, this fact was declared by C.L.Siegel [2] in 1932 only. It seems that first mathematical publication devoted to the calculation of zeta zeros belongs to Gram [3] (1895). He established that $\gamma_{1}=14.135, \gamma_{2}=20.82, \gamma_{3}=25.1$, but his method was too laborious and unfit for the detecting of higher zeros. In 1902, Gram invented a more acceptable method of detecting of the zeros of $\zeta(s)$.

The key idea of this method is the following. Let $A(t)$ and $B(t)$ be a real and imaginary parts of $\zeta\left(\frac{1}{2}+i t\right)$ correspondingly. Then

$$
\zeta\left(\frac{1}{2}+i t\right)=e^{-i \vartheta(t)} Z(t)=Z(t)(\cos \vartheta(t)-i \sin \vartheta(t))
$$

and hence $A(t)=Z(t) \cos \vartheta(t), B(t)=-Z(t) \sin \vartheta(t)$. Now let us consider the real zeros of $B(t)$. These zeros are of two types. The zeros of the first type are the ordinates $\gamma_{n}$ of zeros of $\zeta(s)$ lying on the critical line, and the zeros of the second type are the roots of the equation $\sin \vartheta(t)=0$. Using the Stirling's formula in the form

$$
\vartheta(t)=\frac{t}{2} \ln \frac{t}{2 \pi}-\frac{t}{2}-\frac{\pi}{8}+O\left(\frac{1}{t}\right),
$$

and considering the values $t>7$, it's possible to show that the roots of the above equation generate the unbounded monotonic sequence: $t_{0}=9.6669 \ldots, t_{1}=17.8456 \ldots$, $t_{2}=23.1703 \ldots, t_{3}=27.6702 \ldots, \ldots$ Here $t_{n}$ denotes $n$th Gram point i.e. the unique solution of the equation $\vartheta\left(t_{n}\right)=(n-1) \pi$. Thus, the value $\zeta\left(\frac{1}{2}+i t_{n}\right)$ is real and

$$
\zeta\left(\frac{1}{2}+i t_{n}\right)=A\left(t_{n}\right)=Z\left(t_{n}\right) \cos \pi(n-1)=(-1)^{n-1} Z\left(t_{n}\right) .
$$

Suppose now that $A(t)$ has the same sign at the points $t_{n-1}$ and $t_{n}$ for some $n$. Then the values $Z\left(t_{n-1}\right)$ and $Z\left(t_{n}\right)$ are of opposite sign. Therefore, $Z(t)$ vanishes at the odd number of points between $t_{n-1}$ and $t_{n}$.

Using Euler - Maclaurin summation formula, Gram established that $A\left(t_{n}\right)>0$ for $n=1,2, \ldots, 15$, and proved that all zeros of $\zeta(s)$ in the strip $0<t<66$ lie on the critical line. This method allowed him also to find approximately the ordinates $\gamma_{1}, \gamma_{2}, \ldots, \gamma_{15}$. Thus Gram established that there is exactly one zero $c_{n}$ in each interval $G_{n}=\left(t_{n-1}, t_{n}\right]$, $n=1,2, \ldots, 15$ and, moreover, $t_{n-1}<c_{n}<t_{n}$. However, he assumed also that this law is not universal: ' $\ldots$ the values $A\left(t_{n}\right)$ are positive for all $t_{n}$ lying between 10 and 65 . It seems that the function $A(t)$ is positive for a most part of $t$ under consideration. Obviously, the reason is that the first term of the sum $\sum_{1}^{n} n^{-1 / 2} \cos (t \log n)$ leads to the dominance of positive summands. If it is so, the regularity in the relative location of $c_{n}$ and $t_{n}$ will hold true for some time for the roots c lying closely to $c_{15}$ until the equilibrium will set in (see [1]; for simplicity, we use here the notations of the present paper).

The words 'Gram's law' appeared for the first time in Hutchinson's paper [4]. He used this notion to underline the property of $c_{n}$ and $c_{n+1}$ to be separated by Gram's point $t_{n}$. Hutchinson undertook more wide calculations of zeros of $\zeta(s)$ in order to check the validity of Gram's assumption. He found two first values of $n$ that do not satisfy Gram's law: $n=127$ and $n=136$. Namely, he established that

$$
t_{127}<\gamma_{127}<\gamma_{128}<t_{128}, \quad t_{134}<\gamma_{135}<\gamma_{136}<t_{135} .
$$


Ten years later, E.C.Titchmarsh and L.J.Comrie [5],[6] continued Hutchinson's calculations using Brunsviga, National and Hollerith machines. They found a lot of new exceptions from Gram's law, but the proportion of these exceptions does not exceed $4.5 \%{ }^{2}$. The paper [5] contains also the first theoretical results concerning Gram's law. Thus, Titchmarsh proved that the inequality $A\left(t_{n}\right)=(-1)^{n-1} Z\left(t_{n}\right)>0$ fails for infinitely many $n$. Moreover, he proved that the sequence of fractions $\tau_{n}=\frac{c_{n}-t_{n}}{t_{n+1}-t_{n}}$ is unbounded. The last assertion means that there are infinitely many zeros $c_{n}$ lying outside the interval $G_{n}$.

Though the rule with infinitely many exceptions is not a law in a rigorous sense, the notion 'Gram's law' is widely used now, but sometimes in different senses. We will also use this notion for any assertion concerning the relative location of ordinates of zeros of $\zeta(s)$ and Gram points. In what follows, we give a sort of 'classification' of 'Gram's laws'.

Definition 1. Gram's interval $G_{n}=\left(t_{n-1}, t_{n}\right]$ satisfies to the Strict Gram's Law (SGL) iff $G_{n}$ contains a zero $c_{n}$ of $Z(t)$.

This definition is close to that Hutchinson and Titchmarsh used. But here we allow the coincidence of $t_{n}$ and a zero of Hardy's function. The reason is that now very little is known about the number of such coincidences (or noncoincidences). It seems that $Z\left(t_{n}\right)$ does not vanish for every $n$, i.e. $c_{m} \neq t_{n}$ for any $m$ and $n$. We only know that $Z\left(t_{n}\right) \neq 0$ for at least $(4-o(1)) N(\ln N)^{-1}$ values of $n, 1 \leqslant n \leqslant N(\text { see [7] })^{3)}$. The unboundedness of the fractions $\tau_{n}$ implies that SGL fails for infinitely many cases. Unfortunately, it is still unknown whether the number of cases when SGL holds true is finite or infinite.

The definition 1 contains a very heavy restriction. Namely, the number of the interval $G_{n}$ and the number of zero $c$ belonging to $G_{n}$ must be equal. The renunciation of this restriction leads to the second version of Gram's law.

Definition 2. Gram's interval $G_{n}$ satisfies to Gram's law (GL) iff $G_{n}$ contains exactly one (simple) zero of $Z(t)$.

It's possible to show that SGL and GL are not equivalent to each other. The failure (validity) of one statement does not imply the failure (validity) of another. For example, $G_{1}, \ldots, G_{126}$ satisfy both SGL and GL; $G_{127}$ does not satisfy neither SGL, nor GL; further, $G_{128}$ satisfies SGL, but does not satisfy GL; finally, $G_{3359}, G_{3778}, G_{4542}$ satisfy GL, but do not satisfy SGL.

The counting of zeros of $Z(t)$ in given interval $(a, b)$ usually reduces to the determining of number of sign-changes of $Z(t)$ in $(a, b)$. Therefore, this method allows to determine the evenness of the number of zeros only. For example, the inequality $Z(a) Z(b)<0$ guarantees the existence of an odd number of zeros in $(a, b)$ counted with multiplicity. Therefore, it seems natural to consider one more type of Gram's law.

${ }^{2)}$ Titchmarsh mentioned 43 exceptions that he had found during the calculation of first 1041 zeros of $Z(t)$ lying in the interval $0<t \leqslant 1468$. However, there are 1042 zeros of Hardy's function and 1041 Gram's points $t_{n}$ between $t=0$ and $t=1468$, and there are 45 values of $n$ such that $(-1)^{n-1} Z\left(t_{n}\right)<0$.

${ }^{3)}$ Selberg's theorem formulated without proof in [8] and cited below implies that $Z\left(t_{n}\right) \neq 0$ for positive proportion of $n$. It's interesting to note that the values $Z\left(t_{n}\right)$ are very close to zero for some $n$. Thus, the minima of $\left|Z\left(t_{n}\right)\right|$ for $n \leqslant 10^{5}$ and $n \leqslant 10^{6}$ are equal to $1.238 \cdot 10^{-5}(n=97281)$ and to $8.908 \cdot 10^{-8}$ $(n=368383)$ correspondingly. 
Definition 3. Gram's interval $G_{n}$ satisfies to Weak Gram's Law (WGL) iff $G_{n}$ contains an odd number of zeros of $Z(t)$.

Obviously, GL implies WGL, but the opposite statement is incorrect. Thus, if $n=$ 2147 then $G_{n}$ contains exactly three zeros of Hardy's function, namely $c_{n-1}, c_{n}$ and $c_{n+1}$. The inequality $Z\left(t_{n-1}\right) Z\left(t_{n}\right)<0$ is a sufficient (but not necessary) condition for WGL. Therefore, Titchmarsh's formula (see [9])

$$
\sum_{n \leqslant N} Z\left(t_{n-1}\right) Z\left(t_{n}\right) \sim-2(\gamma+1) N
$$

implies that WGL holds true in infinitely many cases ( $\gamma$ denotes Euler's constant).

The statement 'SGL holds true for all $n \geqslant n_{0}$ ' implies the boundedness of the fractions $\tau_{n}$ as $n \rightarrow+\infty$. The last fact contradicts to some properties of $S(t)$ established by H.Bohr and E.Landau [10] in 1913.

In the middle of 40's, Selberg invented a new powerful method of researching of $S(t)$ (see [11], [12]) and obtained a lot of very deep results concerning the distribution of zeros of $\zeta(s)$. In particular, in [8] he formulated (without proof) the following theorem: there exist absolute constants $K$ and $N_{0}$ such that for $N>N_{0}, 1 \leqslant n \leqslant N$, the numbers $Z\left(t_{n-1}\right)$ and $Z\left(t_{n}\right)$ are of different sign in more than $K N$ cases, and of the same sign in more than $K N$ cases. This theorem implies that both WGL and GL fail for positive proportion of cases, and that WGL holds true for positive proportion of cases.

Denote by $\nu_{k}=\nu_{k}(N)$ the number of Gram's intervals $G_{n}, 1 \leqslant n \leqslant N$, that contain exactly $k$ ordinates of zeros of $\zeta(s)$ (here we consider all the zeros in the critical strip, but not only the zeros lying on the critical line). It's not difficult to prove that Selberg's theorem implies the following relations:

$$
\begin{aligned}
& \nu_{0}+\nu_{2}+\nu_{4}+\ldots \geqslant K N, \\
& \nu_{1}+\nu_{3}+\nu_{5}+\ldots \geqslant K N,
\end{aligned}
$$

These inequalities are weaker than the original Selberg's assertion. The reason is that Selberg's theorem deals with the ordinates in open intervals $\left(t_{n-1}, t_{n}\right)$ and with non-vanishing of $Z(t)$ at the end-points of such intervals for positive proportion of $n$.

Further, (2) implies weaker estimate

$$
\nu_{0}+\nu_{2}+\nu_{3}+\nu_{4}+\ldots \geqslant K N \text {. }
$$

This inequality shows that the positive proportion of Gram's intervals contain an 'abnormal' number (i.e. $\neq 1$ ) of ordinates. Both $(2)$ and $(4)$ imply that WGL and GL fail for positive proportion of $n$.

As far as author knows, the proof of Selberg's theorem or the proof of (2), (3) were never published. The estimate (4) was proved by T.S. Trudgian [14] in 20094). He also pointed out in [14] that (4) implies the inequality

$$
\nu_{0}>K_{1} N
$$

\footnotetext{
${ }^{4)}$ It is necessary to note that the inequalities $\nu_{0} \gg N, \nu_{2}+\nu_{3}+\nu_{4}+\ldots \gg N$ were formulated without proof by A. Fujii in [15]
} 
for any fixed $K_{1}, 0<K_{1}<\frac{1}{2} K$, and for $N \geqslant N_{0}\left(K_{1}\right)$. Indeed, the following identities hold true:

$$
\begin{aligned}
& 0 \cdot \nu_{0}+1 \cdot \nu_{1}+2 \cdot \nu_{2}+3 \cdot \nu_{3}+\ldots=N+S\left(t_{N}+0\right) \\
& \nu_{0}+\nu_{1}+\nu_{2}+\nu_{3}+\ldots=N
\end{aligned}
$$

It's easy to see that (6) expresses the fact that the number of zeros whose ordinates are positive and do not exceed $t_{N}$, is equal to $N\left(t_{N}+0\right)=N+S\left(t_{N}+0\right)$, and (7) expresses the fact that the number of $G_{n}$ containing in $\left(0, t_{N}\right]$ is equal to $N$. Subtracting $(7)$ from (6) we find:

$$
\nu_{0}=\nu_{2}+2 \nu_{3}+3 \nu_{4}+\ldots-S\left(t_{N}+0\right) .
$$

Adding $\nu_{0}$ to both parts and using the classical estimate $S(t)=O(\ln t)$ (see [13]) we get:

$$
\begin{aligned}
2 \nu_{0}=\nu_{0}+\nu_{2}+2 \nu_{3}+3 \nu_{4} & +\ldots \geqslant \\
& \geqslant \nu_{0}+\nu_{2}+n u_{3}+\nu_{4}+\ldots+O(\ln N) \geqslant K N+O(\ln N) .
\end{aligned}
$$

This proves (5). Similarly to (2) and (4), the inequality (5) implies that both WGL and GL fail for positive proportion of cases.

It's interesting to note the following. It is supposed that $\nu_{1}(N) \geqslant c N$ or even $\nu_{1}(N) \sim$ $c_{1} N$ as $N$ growths. However, weaker relation $\nu_{1}(N) \rightarrow+\infty$ as $N \rightarrow+\infty$ is still unproved. Thus, we don't know whether the number of cases when GL holds true is finite or not.

There are some reasons to think that Selberg interpreted Gram's Law in a way different from Titchmarsh's one and different from SGL, GL and WGL. In dealing with Gram's Law, Titchmarsh considered only the real zeros of Hardy's function. We think that Selberg considered all the zeros of $\zeta(s)$ in the critical strip. The basic arguments that sustain this point of view will be introduced later. Now we give here some preliminary notes.

Let $\gamma_{n}$ be an ordinate of any zero of $\zeta(s)$ in the critical strip. Then we determine a unique integer $m=m(n)$ such that $t_{m-1}<\gamma_{n} \leqslant t_{m}$, and set $\Delta_{n}=m-n$.

Definition 4. We say that Gram - Selberg's Phenomenon (GSP) is observed for the ordinate $\gamma_{n}$ iff $\Delta_{n}=0$, i.e. iff $t_{n-1}<\gamma_{n} \leqslant t_{n}$.

It seems very probable that the property of $\gamma_{n}$ to satisfy the condition $\Delta_{n}=0$ was called by Selberg as 'Gram's Law'.

The above result of Selberg implies that there is a positive proportion of cases when GSP does not observed. However, it's possible to say much more about GSP. Thus, Selberg established the formulas

$$
\begin{aligned}
& \sum_{n \leqslant N} \Delta_{n}^{2 k}=\frac{(2 k) !}{k !} \frac{N}{(2 \pi)^{2 k}}(\ln \ln N)^{k}+O\left(N(\ln \ln N)^{k-1 / 2}\right), \\
& \sum_{n \leqslant N} \Delta_{n}^{2 k-1}=O\left(N(\ln \ln N)^{k-1}\right),
\end{aligned}
$$


where $k \geqslant 1$ is a fixed integer, and assumed that the inequalities

$$
\frac{1}{\Phi(n)} \sqrt{\ln \ln n}<\left|\Delta_{n}\right| \leqslant \Phi(n) \sqrt{\ln \ln n}
$$

hold true for 'almost all' $n$. Here $\Phi(x)$ denotes any fixed positive unbounded function. In particular, this assumption implies that GSP does not observed in 'almost all' cases.

It follows from the remark on p.355 of [16] that Selberg found a proof of his own assumption a long before 1989, but he didn't publish it. For the reconstruction of such proof, see author's papers [17],[18].

Thus, we can't expect the occurrence of GSP in positive proportion of cases. The reason is that the definition 4 impose a heavy restriction on $\gamma_{n}$ (the ordinate should belong to the Gram's interval with the same number). Thus, GSP is a very rare phenomenon. It's natural to ask whether GSP occurs in infinitely many cases or no. Since no such results were published, some quantitative statements about the frequency of occurrence of GSP seem to have some interest. Author intends to introduce them in a future paper.

Now we place the results concerning Gram's law in the following table.

\begin{tabular}{|c|c|c|}
\hline & holds true & fails \\
\hline SGL & \multirow[t]{2}{*}{$\begin{array}{l}\text { it's unknown, whether the number of } \\
\text { such cases is finite or no }\end{array}$} & $\begin{array}{l}\text { for infinitely many cases: } \\
\text { - Titchmarsh [5], 1935; }\end{array}$ \\
\hline GL & & $\begin{array}{l}\text { for infinitely many cases: } \\
\text { - Titchmarsh [5], 1935; } \\
\text { for positive proportion of cases: } \\
\text { - Selberg [8], 1946; } \\
\text { - Fujii [15], 1987; } \\
\text { - Trudgian [14], 2009. }\end{array}$ \\
\hline WGL & $\begin{array}{l}\text { for infinitely many cases: } \\
\text { - Titchmarsh [9], 1934; } \\
\text { for positive proportion of cases: } \\
\text { - Selberg [8], 1946; }\end{array}$ & $\begin{array}{l}\text { for positive proportion of cases: } \\
\text { - Selberg [8], 1946; } \\
\text { - Fujii [15], 1987; } \\
\text { - Trudgian [14], } 2009 .\end{array}$ \\
\hline GSP & for infinitely many cases & $\begin{array}{l}\text { for infinitely many cases: } \\
\text { - Titchmarsh [5], 1935; } \\
\text { for 'almost all' cases: } \\
\text { - Selberg [16], } 1989 .\end{array}$ \\
\hline
\end{tabular}

The present paper contains some new statements concerning the behavior of the function $S(t)$ at Gram points. These statements are applied to the proof of Selberg's formulas (8), (9) and to other problems connected with Gram's law. The paper is organized as follows.

First, $\S 2$ contains auxiliary assertions. In $§ 3$, the sum

$$
\sum_{N<n \leqslant N+M}\left(S\left(t_{n+m}+0\right)-S\left(t_{n}+0\right)\right)^{2 k}
$$


is calculated. Here $k \geqslant 0, m>0$ are sufficiently large integers, that may growth slowly with $N$ (Theorem 1). The correct bound (in the sense of order of growth) for the sum (10) with $m=1$ is also given here (Theorem 2). It is necessary to note that the bounds of such type are contained in [15]. But they hold true only for a 'long' interval of summation: $1 \leqslant n \leqslant N$ or $N<n \leqslant N+M, M \asymp N$. The statements of present paper are valid for a 'short' interval, namely for the case $M \asymp N^{\alpha+\varepsilon}, \alpha=\frac{27}{82}=\frac{1}{3}-\frac{1}{246}$.

Further, Theorem 3 in $\S 3$ gives a true order of magnitude of the sum

$$
\sum_{N<n \leqslant N+M}\left|S\left(t_{n}+0\right)-S\left(t_{n-1}+0\right)\right| .
$$

This statement is based on Theorems 1 and 2 and plays the key role in the proof of the inequalities

$$
\nu_{0}(N+M)-\nu_{0}(N) \gg M, \quad \sum_{k \geqslant 2}\left(\nu_{k}(N+M)-\nu_{k}(N)\right) \gg M
$$

(Theorem 4). The analogues of these bounds for the case of the 'long' intervals of summation were formulated (without proof) for the first time by Selberg in [8] as a corollary of his theorem cited above.

In $\S 4$, a non-trivial bound for the alternating sum

$$
T_{k}=\sum_{N<n \leqslant N+M} S^{k}\left(t_{n}+0\right)\left(S\left(t_{n}+0\right)-S\left(t_{n-1}+0\right)\right)
$$

is given (Theorem 5). This estimation leads to a new proof of Selberg's formulas (8), (9) (see Theorem 6 ) and of the assumption that $\Delta_{n} \neq 0$ for 'almost all' $n$. The proofs of these facts differ from those given in previous author's paper [18]. They don't use the information about the number of solutions of the inequalities $a<\Delta_{n} \leqslant b$ with the condition $N<n \leqslant N+M$. It's possible that the below proof of Theorem 6 is close to the original Selberg's proof.

Finally, in $\S 6$ we argue that Selberg considered all the complex zeros of $\zeta(s)$ in dealing with the Gram's law in [8]. This question leads us to a new equivalent of 'almost Riemann hypothesis' (see Theorem 7; 'almost Riemann hypothesis' claims that almost all complex zeros of $\zeta(s)$ lie on the critical line).

Throughout the paper, $\varepsilon$ denotes an arbitrary small positive number, $0<\varepsilon<10^{-3}$; $N_{0}(T)$ denotes the number of zeros of $\zeta\left(\frac{1}{2}+i t\right)$ for $0<t \leqslant T ; N \geqslant N_{1}(\varepsilon)>0$ is a sufficiently large integer; $L=\ln \ln N, M$ is an arbitrary integer with the conditions $N^{\alpha+\varepsilon_{1}} \leqslant M \leqslant N^{\alpha+\varepsilon}, \alpha=\frac{27}{82}, \varepsilon_{1}=0.9 \varepsilon ; \theta, \theta_{1}, \theta_{2}, \ldots$ are complex numbers whose absolute values do not exceed 1 and which are, generally speaking, different in different relations. In some cases we use for brevity the notation $\Delta(n)$ for the value $S\left(t_{n}+0\right)$.

\section{$\S$ 2. Auxiliary lemmas}

LEMMA 1. The following relations hold true for any $x \geqslant 2$ :

$$
\sum_{p \leqslant x} \frac{\ln p}{p}<\ln x, \quad \sum_{p \leqslant x} \frac{1}{p}=\ln \ln x+c+\frac{\theta}{\ln ^{2} x} ;
$$


here $c=0.26 \ldots$ and $-\frac{1}{2}<\theta<1$.

For a proof, see [19].

Lemma 2. Suppose that $0<\kappa<\frac{1}{2}, 0<c<\frac{1}{2}-\kappa, \mu, \nu$ are integers such that $\mu, \nu \geqslant 0, \mu+\nu=2 k, k \geqslant 1, N \geqslant \exp \left(9 \kappa^{-1}\right), M \geqslant \exp \left(3 k c^{-1}\right), y=M^{c / k}$. Furthermore, let $p_{1}, \ldots, p_{\nu}, q_{1}, \ldots, q_{\mu}$ take values of prime numbers in the interval $(1, y]$ and satisfy the condition $p_{1} \ldots p_{\nu} \neq q_{1} \ldots q_{\mu}$. Finally, suppose that $|a(p)| \leqslant \delta$ for $p \leqslant y$. Then the sum $S$,

$$
S=\sum_{N<n \leqslant N+M} \sum_{\substack{p_{1}, \ldots, p_{\nu} \\ q_{1}, \ldots, q_{\mu}}} \frac{a\left(p_{1}\right) \ldots a\left(p_{\nu}\right) \bar{a}\left(q_{1}\right) \ldots \bar{a}\left(q_{\mu}\right)}{p_{1} \ldots q_{\mu}}\left(\frac{q_{1} \ldots q_{\mu}}{p_{1} \ldots p_{\nu}}\right)^{i t_{n}}
$$

satisfies the bound $|S|<\left(\delta y^{3 / 2}\right)^{2 k} \ln N$.

For a proof, see [17].

LEMMA 3. Let $k \geqslant 1$ be an integer, $y>e^{3}$, and let $p_{1}, \ldots, p_{k}, q_{1}, \ldots, q_{k}$ take values of prime numbers in the interval $(1, y]$. Then the following relation holds:

$$
\sum_{p_{1} \ldots p_{k}=q_{1} \ldots q_{k}} \frac{a\left(p_{1}\right) \ldots a\left(p_{k}\right) \bar{a}\left(q_{1}\right) \ldots \bar{a}\left(q_{k}\right)}{\sqrt{p_{1} \ldots q_{k}}}=k !\left(\sigma_{1}^{k}+\theta_{k} k^{2} \sigma_{1}^{k-2} \sigma_{2}\right),
$$

where

$$
\sigma_{j}=\sum_{p \leqslant y}\left(\frac{|a(p)|^{2}}{p}\right)^{j}, \quad j=1,2, \quad-1 \leqslant \theta_{k} \leqslant 0
$$

and $\theta_{1}=0$.

For a proof, see [20],[21].

Suppose $x=t_{N}^{0.1 \varepsilon}$. For positive $t$ and $y$ we define

$$
V(t)=V_{y}(t)=\frac{1}{\pi} \sum_{p<y} \frac{\sin (t \ln p)}{\sqrt{p}}, \quad R(t)=S(t)+V(t) .
$$
Then

LEMMA 4. Suppose $k$ is an integer such that $1 \leqslant k \leqslant \frac{1}{192} \ln x, y=x^{1 /(4 k)}, V(t)=V_{y}(t)$.

$$
\sum_{N<n \leqslant N+M} R^{2 k}\left(t_{n}+0\right) \leqslant\left(A e^{-4} k\right)^{2 k} M
$$

where $A=e^{21} \varepsilon^{-1.5}$.

Lemma 5. Suppose $k$ is an integer, $1 \leqslant k \leqslant \sqrt{L}$. Then the following relations hold:

$$
\begin{aligned}
& \sum_{N<n \leqslant N+M} S^{2 k}\left(t_{n}+0\right)=\frac{(2 k) !}{k !} \frac{M L^{k}}{(2 \pi)^{2 k}}\left(1+\theta A^{k} L^{-0.5}\right), \\
& \left|\sum_{N<n \leqslant N+M} S^{2 k-1}\left(t_{n}+0\right)\right| \leqslant \frac{3.5}{\sqrt{B}}(B k)^{k} M L^{k-1}, \\
& \sum_{N<n \leqslant N+M} S^{2 k}\left(t_{n}+0\right) \leqslant 2\left(\frac{k A}{\pi^{2} e}\right)^{k} M L^{k},
\end{aligned}
$$


where $B=A^{2} e^{-8}$, and $A$ is defined in Lemma 4 .

For the proofs of these two lemmas, see [17] (the substitution of $t_{n}$ to $t_{n}+0$ does not affect the truth of the result; the reason is that the above substitution does not affect to the functions that approximate $S(t)$ in the proofs of Lemmas 4 and 5).

Lemma 6. Suppose $m$ is an integer, $1 \leqslant m \leqslant M$. Then the equality

$$
t_{n+m}-t_{n}=\frac{\pi m}{\vartheta^{\prime}\left(t_{N}\right)}+\frac{3 \theta M}{N(\ln N)^{2}}
$$

holds true for $N<n \leqslant N+M$.

Proof. By Lagrange's mean value theorem, we have

$$
\pi m=\vartheta\left(t_{n+m}\right)-\vartheta\left(t_{n}\right)=\left(t_{n+m}-t_{n}\right) \vartheta^{\prime}(\xi), \quad t_{n+m}-t_{n}=\frac{\pi m}{\vartheta^{\prime}(\xi)}
$$

for some $\xi, t_{n}<\xi<t_{n+m}$. Since $\vartheta^{\prime}(t), \vartheta^{\prime \prime}(t)$ are monotonic for $t>7$, by the inequality

$$
\frac{t_{N}}{2 \pi} \ln \frac{t_{N}}{2 \pi}>N
$$

we get:

$$
\begin{aligned}
0<\frac{1}{\vartheta^{\prime}\left(t_{N}\right)}- & \frac{1}{\vartheta^{\prime}(\xi)}=\frac{\vartheta^{\prime}(\xi)-\vartheta^{\prime}\left(t_{N}\right)}{\vartheta^{\prime}\left(t_{N}\right) \vartheta^{\prime}(\xi)} \leqslant \\
& \leqslant \frac{\left(t_{N+M}-t_{N}\right) \vartheta^{\prime \prime}\left(t_{N}\right)}{\left(\vartheta^{\prime}\left(t_{N}\right)\right)^{2}}=\frac{\pi M}{\vartheta^{\prime}(\zeta)} \frac{\vartheta^{\prime \prime}\left(t_{N}\right)}{\left(\vartheta^{\prime}\left(t_{N}\right)\right)^{2}}<\frac{\pi M \vartheta^{\prime \prime}\left(t_{N}\right)}{\left(\vartheta^{\prime}\left(t_{N}\right)\right)^{3}}<\frac{3 M}{N(\ln N)^{2}} .
\end{aligned}
$$

This proves the lemma.

Lemma 7. Suppose $0<h_{0}<\frac{1}{2}$ is a sufficientlty small constant, $0<h<h_{0}$, $h \ln x>2$, and let

$$
V(x ; h)=\sum_{p \leqslant x} \frac{\sin ^{2}\left(\frac{1}{2} h \ln p\right)}{p} .
$$

Then the following relation holds:

$$
V(x ; h)=\frac{1}{2} \ln (h \ln x)+1.05 \theta .
$$

ProOF. Setting $y=e^{\lambda / h}$ for some $1<\lambda<2$, we obtain:

$$
\begin{aligned}
V(x ; h)=\left(\sum_{p \leqslant y}+\right. & \left.\sum_{y<p \leqslant x}\right) \frac{\sin ^{2}\left(\frac{1}{2} h \ln p\right)}{p}= \\
& =\sum_{p \leqslant y} \frac{\sin ^{2}\left(\frac{1}{2} h \ln p\right)}{p}+\frac{1}{2} \sum_{y<p \leqslant x} \frac{1-\cos (h \ln p)}{p}=V_{1}+\frac{1}{2}\left(V_{2}-V_{3}\right) .
\end{aligned}
$$


The application of Lemma 1 yields:

$$
\begin{aligned}
V_{1} & \leqslant\left(\frac{h}{2}\right)^{2} \sum_{p \leqslant y} \frac{(\ln p)^{2}}{p} \leqslant\left(\frac{h}{2}\right)^{2}(\ln y) \sum_{p \leqslant y} \frac{\ln p}{p}<\left(\frac{h}{2} \ln y\right)^{2}=\frac{\lambda^{2}}{4}, \\
V_{2} & =\left(\sum_{p \leqslant x}-\sum_{p \leqslant y}\right) \frac{1}{p}=\ln (h \ln x)-\ln \lambda+2 \theta\left(\frac{h}{\lambda}\right)^{2} .
\end{aligned}
$$

Now we divide the domain of $p$ in $V_{3}$ into the intervals of the form $a<p \leqslant b$, where $b \leqslant 2 a, a=2^{k} y, k=0,1,2, \ldots$. Thus we get:

$$
V_{3}=\operatorname{Re} \sum_{y<p \leqslant x} \frac{p^{i h}}{p}=\operatorname{Re} \sum_{a} V_{3}(a), \quad V_{3}(a)=\sum_{a<p \leqslant b} \frac{p^{i h}}{p} .
$$

Setting

$$
\mathbb{C}(u)=\sum_{a<p \leqslant u} \frac{1}{p}=\ln \ln u-\ln \ln a+\frac{2 \theta}{\ln ^{2} a}
$$

and applying the Abel's summation formula, we have

$$
V_{3}(a)=\mathbb{C}(b) b^{i h}-\int_{a}^{b} \mathbb{C}(u) d u^{i h}=\int_{a}^{b} \frac{u^{i h} d u}{u \ln u}+\frac{3 \theta_{1}}{\ln ^{2} a},
$$

and therefore

$$
V_{3}=\operatorname{Re}(j)+3 \theta_{2} \sum_{k \geqslant 0} \frac{1}{(k \ln 2+\ln y)^{2}}, \quad j=\int_{y}^{x} \frac{u^{i h} d u}{u \ln u} .
$$

Integration by parts yields:

$$
j=\frac{1}{i h}\left(\frac{x^{i h}}{\ln x}-\frac{y^{i h}}{\ln y}-\int_{y}^{x} u^{i h} d \frac{1}{\ln u}\right), \quad|j| \leqslant \frac{2}{h \ln y}=\frac{2}{\lambda} .
$$

Finally, we obtain

$$
\begin{aligned}
& \left|V_{3}\right| \leqslant \frac{2}{\lambda}+\frac{9}{\ln y}=\frac{2+9 h}{\lambda}, \\
& V(x ; h)=\frac{1}{2} \ln (h \ln x)+v(x ; h),
\end{aligned}
$$

where

$$
|v(x ; h)| \leqslant \frac{\lambda^{2}}{4}+\frac{1}{\lambda}+\frac{1}{2} \ln \lambda+\frac{9 h}{\lambda}+\frac{h^{2}}{\lambda^{2}} .
$$

Setting $\lambda=1.5$, we arrive at the assertion of the lemma.

Lemma 8. $N_{0}(t)>\left(0.4+7 \cdot 10^{-3}\right) N(t)$ for $t>t_{0}>1$.

For a proof, see [22]. 


\section{§3. On mean values of the quantities $S\left(t_{n+m}+0\right)-S\left(t_{n}+0\right)$}

Suppose $m \geqslant 1$ is an integer. Let us consider the union of $m$ adjacent Gram's intervals $G_{n+1}, G_{n+2}, \ldots, G_{n+m}$, that is the interval $\left(t_{n}, t_{n+m}\right]$. By Riemann - von Mangoldt's formula (1), the number of ordinates in last interval equals to

$$
\begin{array}{r}
N\left(t_{n+m}+0\right)-N\left(t_{n}+0\right)=\frac{1}{\pi}\left(\vartheta\left(t_{n+m}\right)-\vartheta\left(t_{n}\right)\right)+S\left(t_{n+m}+0\right)-S\left(t_{n}+0\right)= \\
=m+S\left(t_{n+m}+0\right)-S\left(t_{n}+0\right) .
\end{array}
$$

The number of $\gamma_{n}$ that do not exceed a given bound equals asymptotically to the number of Gram points in the same domain. Then it is natural to call the number $m$ as an 'expected' number of ordinates of zeros of $\zeta(s)$ in the interval $\left(t_{n}, t_{n+m}\right]$. Hence, the difference

$$
S\left(t_{n+m}+0\right)-S\left(t_{n}+0\right)
$$

is a deviation of 'true' number of ordinates from the 'expected' number.

The below Theorem 1 shows that this deviation often takes a very large values (of order $\sqrt{\ln m}$, for example). This fact was observed firstly by Fujii [15] for the case when the interval of summation is long $(1 \leqslant n \leqslant N)$ and when $m$ growths with $N$. He proved that the distribution function for the normalized differences (12) tends to Gaussian distribution as $N \rightarrow+\infty$.

THEOREM 1. Let $k$ and $m$ be an integers that satisfy the conditions

$$
k \geqslant 1, \quad k \varepsilon^{-1} \exp \left(\lambda k^{2}\right) \leqslant m \leqslant c \ln N,
$$

where $\lambda=\left(2 B e \pi^{2}\right)^{2}, B$ is defined in Lemma 5 , and $c$ is a sufficiently small absolute constant. Then the following relation holds:

$$
\sum_{N<n \leqslant N+M}\left(S\left(t_{n+m}+0\right)-S\left(t_{n}+0\right)\right)^{2 k}=\frac{(2 k) !}{k !} M\left(\frac{1}{2 \pi^{2}} \ln \frac{m \varepsilon}{k}\right)^{k}\left(1+\frac{6 \theta \sqrt{B} 4^{k} k \sqrt{k}}{\sqrt{\ln (m \varepsilon / k)}}\right) .
$$

Proof. Let $x=t_{N}^{0.1 \varepsilon}, y=x^{1 /(4 k)}, V(t)=V_{y}(t)$. By lemma $6, t_{n+m}-t_{n}=h+\varepsilon_{n}$, where

$$
h=\frac{\pi m}{\vartheta^{\prime}\left(t_{N}\right)}, \quad\left|\varepsilon_{n}\right| \leqslant \frac{3 M}{N(\ln N)^{2}} .
$$

By Lagrange's mean value theorem and the inequalities

$$
\left|V^{\prime}(t)\right| \leqslant \frac{1}{\pi} \sum_{p<y} \frac{\ln p}{\sqrt{p}}<\sqrt{y}=x^{1 /(8 k)}<N^{\varepsilon / 80}
$$

we get

$V\left(t_{n+m}\right)=V\left(t_{n}+h+\varepsilon_{n}\right)=V\left(t_{n}+h\right)+\varepsilon_{n} V^{\prime}\left(t_{n}+h+\theta \varepsilon_{n}\right)=V\left(t_{n}+h\right)+\theta_{1} N^{-2 / 3}$. 
Hence

$$
\begin{aligned}
& V\left(t_{n+m}\right)-V\left(t_{n}\right)=\frac{2}{\pi} W\left(t_{n}\right)+\theta_{2} N^{-2 / 3}, \\
& W(t)=\frac{1}{2} \sum_{p<y} \frac{\sin ((t+h) \ln p)-\sin (t \ln p)}{\sqrt{p}}=\sum_{p<y} \frac{\sin \left(\frac{1}{2} h \ln p\right)}{\sqrt{p}} \cos \left(\left(t+\frac{1}{2} h\right) \ln p\right) .
\end{aligned}
$$

Using the trivial bound $|W(t)|<\sqrt{y}$ and Lagrange's mean value theorem, we obtain

$$
\begin{aligned}
& \left(V\left(t_{n+m}\right)-V\left(t_{n}\right)\right)^{2 k}=\left(\frac{2}{\pi}\right)^{2 k} W^{2 k}\left(t_{n}\right)+\theta k 2^{2 k-1}\left(\left|W\left(t_{n}\right)\right|^{2 k-1} N^{-2 / 3}+N^{-4 k / 3}\right)= \\
& =\left(\frac{2}{\pi}\right)^{2 k} W^{2 k}\left(t_{n}\right)+\theta_{1} x N^{-2 / 3} .
\end{aligned}
$$

By summing over $n$ and denoting the corresponding sum by $W_{1}$, we have

$$
W_{1}=\sum_{N<n \leqslant N+M}\left(V\left(t_{n+m}\right)-V\left(t_{n}\right)\right)^{2 k}=\left(\frac{2}{\pi}\right)^{2 k} W_{2}+\theta_{2} N^{-1 / 3},
$$

where

$$
W_{2}=\sum_{N<n \leqslant N+M} W^{2 k}\left(t_{n}\right) .
$$

Next, we write $W(t)=\frac{1}{2}(U(t)+\bar{U}(t))$, where

$$
U(t)=\sum_{p<y} \frac{a(p)}{\sqrt{p}} p^{i t}, \quad a(p)=p^{i h / 2} \sin \left(\frac{1}{2} h \ln p\right) .
$$

Then

$$
\begin{aligned}
W_{2} & =2^{-2 k} \sum_{\nu=0}^{2 k}\left(\begin{array}{c}
2 k \\
\nu
\end{array}\right) w_{\nu}, \\
w_{\nu} & =\sum_{N<n \leqslant N+M} \sum_{\substack{p_{1}, \ldots, p_{\nu}<y \\
q_{1}, \ldots, q_{\mu}<y}} \frac{a\left(p_{1}\right) \ldots a\left(p_{\nu}\right) \bar{a}\left(q_{1}\right) \ldots \bar{a}\left(q_{\mu}\right)}{\sqrt{p_{1} \ldots q_{\mu}}}\left(\frac{p_{1} \ldots p_{\nu}}{q_{1} \ldots q_{\mu}}\right)^{i t_{n}},
\end{aligned}
$$

where $\mu=2 k-\nu$. By setting $\kappa=\frac{1}{4}, c=(k \ln y)(\ln M)^{-1}, \delta=1$ in Lemma 2, we obviously have $0<c<\frac{1}{4}=\frac{1}{2}-\kappa, N \geqslant e^{36}=\exp \left(9 \kappa^{-1}\right), y=x^{1 /(4 k)}>e^{4}$,

$$
\exp \left(3 k c^{-1}\right)=\exp \left(\frac{3 \ln M}{\ln y}\right) \leqslant \exp \left(\frac{3}{4} \ln M\right)<M .
$$

Thus, the conditions of Lemma 2 are satisfied. Hence, for $\nu \neq k$ we have

$$
\left|w_{\nu}\right| \leqslant\left(y^{3 / 2}\right)^{2 k} \ln N=x^{3 / 4} \ln N<x .
$$


The contribution of the terms of $w_{k}$ that obey the condition $p_{1} \ldots p_{k} \neq q_{1} \ldots q_{k}$ is estimated as above. Therefore,

$$
W_{2}=2^{-2 k}\left(\begin{array}{c}
2 k \\
k
\end{array}\right) M w+\theta 2^{-2 k} \sum_{\nu=0}^{2 k}\left(\begin{array}{c}
2 k \\
\nu
\end{array}\right) x=2^{-2 k}\left(\begin{array}{c}
2 k \\
k
\end{array}\right) M w+\theta x,
$$

where

$$
w=\sum_{p_{1} \ldots p_{k}=q_{1} \ldots q_{k}} \frac{a\left(p_{1}\right) \ldots \bar{a}\left(q_{k}\right)}{\sqrt{p_{1} \ldots q_{k}}} .
$$

By Lemma $3, w=k !\left(\sigma_{1}^{k}+\theta_{k} k^{2} \sigma_{1}^{k-2} \sigma_{2}\right)$, where

$$
\sigma_{1}=\sum_{p<y} \frac{\sin ^{2}\left(\frac{1}{2} h \ln p\right)}{p}, \quad \sigma_{2} \leqslant \sum_{p} \frac{1}{p^{2}}<\frac{1}{2}
$$

and $-1 \leqslant \theta_{k} \leqslant 0$. Since

$$
\begin{aligned}
& h \ln y=\frac{2 \pi m \ln y}{\ln t_{N}}(1+o(1))=\frac{\pi m \varepsilon}{20 k}(1+o(1))>2, \\
& h \leqslant \frac{2 \pi m}{\ln N}(1+o(1)) \leqslant 2 \pi c(1+o(1)),
\end{aligned}
$$

the conditions of lemma 7 are satisfied for $h_{0}=7 c$ and for sufficiently small $c$. Hence,

$$
\sigma_{1}=\frac{1}{2} \ln (h \ln y)+1.05 \theta=\frac{1}{2} \ln \frac{\pi m \varepsilon}{20 k}(1+o(1))+1.05 \theta=\frac{1}{2} \ln \frac{m \varepsilon}{k}+2 \theta_{1} .
$$

Further, the inequality $\ln (m \varepsilon / k) \geqslant 100 k$ implies the following bounds for $\sigma_{1}, w, W_{2}$ and $W_{1}$ :

$$
\begin{aligned}
& \sigma_{1}^{k} \leqslant\left(\frac{1}{2} \ln \frac{m \varepsilon}{k}\right)^{k}\left(1+\frac{4}{\ln (m \varepsilon / k)}\right)^{k} \leqslant\left(\frac{1}{2} \ln \frac{m \varepsilon}{k}\right)^{k}\left(1+\frac{1}{25 k}\right)^{k}<1.1\left(\frac{1}{2} \ln \frac{m \varepsilon}{k}\right)^{k}, \\
& w \leqslant k ! \sigma_{1}^{k}<1.1 k !\left(\frac{1}{2} \ln \frac{m \varepsilon}{k}\right)^{k}, \\
& W_{2} \leqslant 2^{-2 k} \frac{(2 k) !}{k !} M \cdot 1.1\left(\frac{1}{2} \ln \frac{m \varepsilon}{k}\right)^{k}+x, \\
& W_{1} \leqslant \pi^{-2 k} \frac{(2 k) !}{k !} M \cdot 1.1\left(\frac{1}{2} \ln \frac{m \varepsilon}{k}\right)^{k}+x+N^{-1 / 3}<2\left(\frac{2 k}{\pi^{2} e}\right)^{k}\left(\ln \frac{m \varepsilon}{k}\right)^{k} M .
\end{aligned}
$$

Moreover,

$$
\begin{aligned}
& w=k !\left(\left(\frac{1}{2} \ln \frac{m \varepsilon}{k}+2 \theta\right)^{k}+\theta_{k} k^{2}\left(\frac{1}{2} \ln \frac{m \varepsilon}{k}+2\right)^{k-2}\right)= \\
& =k !\left(\left(\frac{1}{2} \ln \frac{m \varepsilon}{k}\right)^{k}+2 \theta k\left(\frac{1}{2} \ln \frac{m \varepsilon}{k}+2\right)^{k-1}+\theta_{k} k^{2}\left(\frac{1}{2} \ln \frac{m \varepsilon}{k}+2\right)^{k-2}\right)=
\end{aligned}
$$




$$
\begin{aligned}
& =k !\left(\left(\frac{1}{2} \ln \frac{m \varepsilon}{k}\right)^{k}+4 \theta k\left(\frac{1}{2} \ln \frac{m \varepsilon}{k}+2\right)^{k-1}\right), \\
& W_{1}=\left(\frac{2}{\pi}\right)^{2 k}\left(2^{-2 k}\left(\begin{array}{c}
2 k \\
k
\end{array}\right) k ! M\left(\left(\frac{1}{2} \ln \frac{m \varepsilon}{k}\right)^{k}+4 \theta k\left(\frac{1}{2} \ln \frac{m \varepsilon}{k}+2\right)^{k-1}\right)+\theta x\right)+\theta N^{-1 / 3} \\
& =\frac{(2 k) !}{k !} \frac{M}{\pi^{2 k}}\left(\left(\frac{1}{2} \ln \frac{m \varepsilon}{k}\right)^{k}+4.1 \theta k\left(\frac{1}{2} \ln \frac{m \varepsilon}{k}+2\right)^{k-1}\right) .
\end{aligned}
$$

Denoting by $W_{0}$ the initial sum of Lemma and noting that

$$
S\left(t_{n+m}+0\right)-S\left(t_{n}+0\right)=-\left(V\left(t_{n+m}\right)-V\left(t_{n}\right)\right)+\left(R\left(t_{n+m}+0\right)-R\left(t_{n}+0\right)\right),
$$

we get

$$
\begin{aligned}
& \left(S\left(t_{n+m}+0\right)-S\left(t_{n}+0\right)\right)^{2 k}=\left(V\left(t_{n+m}\right)-V\left(t_{n}\right)\right)^{2 k}+ \\
& +\theta k 2^{2 k-1}\left(\left(R\left(t_{n+m}+0\right)-R\left(t_{n}+0\right)\right)^{2 k}+\left|V\left(t_{n+m}\right)-V\left(t_{n}\right)\right|^{2 k-1}\left|R\left(t_{n+m}+0\right)-R\left(t_{n}+0\right)\right|\right), \\
& W_{0}=\sum_{N<n \leqslant N+M}\left(S\left(t_{n+m}+0\right)-S\left(t_{n}+0\right)\right)^{2 k}=W_{1}+\theta_{1} k 2^{2 k-1}\left(W_{3}+W_{4}\right),
\end{aligned}
$$

where

$$
\begin{aligned}
& W_{3}=\sum_{N<n \leqslant N+M}\left(R\left(t_{n+m}+0\right)-R\left(t_{n}+0\right)\right)^{2 k}, \\
& W_{4}=\sum_{N<n \leqslant N+M}\left|V\left(t_{n+m}\right)-V\left(t_{n}\right)\right|^{2 k-1}\left|R\left(t_{n+m}+0\right)-R\left(t_{n}+0\right)\right| .
\end{aligned}
$$

By lemma 4,

$$
\begin{array}{r}
W_{3} \leqslant 2^{2 k-1} \sum_{N<n \leqslant N+M}\left(R^{2 k}\left(t_{n+m}+0\right)+R^{2 k}\left(t_{n}+0\right)\right) \leqslant 2^{2 k} \sum_{N<n \leqslant N+2 M} R^{2 k}\left(t_{n}+0\right) \leqslant \\
\leqslant 2^{2 k} \cdot 2 M(\sqrt{B} k)^{2 k}=2 M(2 \sqrt{B} k)^{2 k} .
\end{array}
$$

Further, combining the above bounds for $W_{1}$ and $W_{3}$ with Hölder's inequality, we have

$$
\begin{aligned}
W_{4} \leqslant W_{1}^{1-1 /(2 k)} W_{3}^{1 /(2 k)} \leqslant 2^{1 /(2 k)} 2 \sqrt{B} k \cdot 2^{1-1 /(2 k)}\left(\frac{2 k}{\pi^{2} e} \ln \frac{m \varepsilon}{k}\right)^{k-1 / 2} M & = \\
& =4 \sqrt{B} k\left(\frac{2 k}{\pi^{2} e} \ln \frac{m \varepsilon}{k}\right)^{k-1 / 2} M .
\end{aligned}
$$

Therefore,

$$
\begin{array}{rl}
k 2^{2 k-1}\left(W_{3}+W_{4}\right) \leqslant k 2^{2 k-1} \cdot 4 & M k \sqrt{B}\left(\frac{2 k}{\pi^{2} e} \ln \frac{m \varepsilon}{k}\right)^{k-1 / 2} M \times \\
\left(1+\left(\frac{k \sqrt{\lambda}}{\ln (m \varepsilon / k)}\right)^{k-1 / 2}\right)<4.1 M k^{2} \sqrt{B}\left(\frac{8 k}{\pi^{2} e} \ln \frac{m \varepsilon}{k}\right)^{k-1 / 2} .
\end{array}
$$


Finally, we get

$$
\begin{aligned}
& W_{0}=\frac{(2 k) !}{k !} \frac{M}{\pi^{2 k}}\left(\left(\frac{1}{2} \ln \frac{m \varepsilon}{k}\right)^{k}+4.1 k \theta_{1}\left(\frac{1}{2} \ln \frac{m \varepsilon}{k}+2\right)^{k-1}\right)+ \\
& +4.1 \theta_{2} \sqrt{B} k^{2}\left(\frac{8 k}{\pi^{2} e} \ln \frac{m \varepsilon}{k}\right)^{k-1 / 2} M=\frac{(2 k) !}{k !} M\left(\frac{1}{2 \pi^{2}} \ln \frac{m \varepsilon}{k}\right)^{k}\left(1+\theta\left(\delta_{1}+\delta_{2}\right)\right),
\end{aligned}
$$

where

$$
\begin{aligned}
& \delta_{1}=4.1 k\left(1+\frac{4}{\ln (m \varepsilon / k)}\right)^{k-1} \frac{2}{\ln (m \varepsilon / k)}<\frac{8.6 k}{\ln (m \varepsilon / k)}, \\
& \delta_{2}=\frac{\pi^{2 k} k !}{(2 k) !} \frac{4.1 \sqrt{B} k^{2}}{\left(\frac{1}{2} \ln (m \varepsilon / k)\right)^{k}}\left(\frac{8 k}{\pi^{2} e} \ln \frac{m \varepsilon}{k}\right)^{k-1 / 2} \leqslant 5.8 \sqrt{B} \frac{4^{k} k \sqrt{k}}{\sqrt{\ln (m \varepsilon / k)}} .
\end{aligned}
$$

It remains to note that

$$
\delta_{1}+\delta_{2}<6 \sqrt{B} \frac{4^{k} k \sqrt{k}}{\sqrt{\ln (m \varepsilon / k)}} .
$$

Thus the theorem is proved.

COROLlary. Suppose $k$ and $m$ are integers such that

$$
1 \leqslant k \leqslant 0.1 \ln \ln \ln N, \quad k \varepsilon^{-1} \exp (\varkappa)<m \leqslant c \ln N,
$$

where $c$ is sufficiently small absolute constant, and $\varkappa$ is a maximum of the numbers $\left(2 B e \pi^{2} k\right)^{2}$ and $12^{2} B 4^{2 k} k^{3}$. Then the following inequality holds:

$$
\sum_{N<n \leqslant N+M}\left(S\left(t_{n+m}+0\right)-S\left(t_{n}+0\right)\right)^{2 k}>\frac{M}{2} \frac{(2 k) !}{k !}\left(\frac{1}{2 \pi^{2}} \ln \frac{m \varepsilon}{k}\right)^{k} .
$$

In particular, if $\varepsilon^{-1} \exp \left(\left(2 B e \pi^{2}\right)^{2}\right)<m \leqslant c \ln N$, then

$$
\sum_{N<n \leqslant N+M}\left(S\left(t_{n+m}+0\right)-S\left(t_{n}+0\right)\right)^{2}>\frac{M}{2 \pi^{2}} \ln (m \varepsilon),
$$

and if $m=[\mu]+1, \mu=\varepsilon^{-1} \exp \left(e^{76} \varepsilon^{-6}\right)$, then

$$
\sum_{N<n \leqslant N+M}\left(S\left(t_{n+m}+0\right)-S\left(t_{n}+0\right)\right)^{2}>1.01 e^{73} \varepsilon^{-6} M .
$$

THeOREM 2. Let $k$ be an integer such that $1 \leqslant k \leqslant \frac{1}{192} \ln x$. Then the following inequality holds:

$$
\sum_{N<n \leqslant N+M}\left(S\left(t_{n}+0\right)-S\left(t_{n-1}+0\right)\right)^{2 k} \leqslant 2 M k(4 k \sqrt{B})^{2 k} .
$$


Proof. Using the same arguments as above, in the case $m=1$ we obtain

$$
W_{1}=\sum_{N<n \leqslant N+M}\left(V\left(t_{n}\right)-V\left(t_{n-1}\right)\right)^{2 k} \leqslant \pi^{-2 k} \frac{(2 k) !}{k !} M \sigma_{1}^{k}+x,
$$

where

$$
\sigma_{1}=\sum_{p<y} \frac{\sin ^{2}\left(\frac{1}{2} h \ln p\right)}{p}, \quad h=\frac{\pi}{\vartheta^{\prime}\left(t_{N}\right)}
$$

Using the relation

$$
\frac{h}{2} \ln y=\frac{\pi}{\ln t_{N}+O(1)} \frac{\ln x}{4 k}<\frac{\ln x}{k \ln t_{N}}<\frac{\varepsilon}{10 k},
$$

and applying Lemma 1, we get

$$
\begin{aligned}
& \sigma_{1} \leqslant \frac{h^{2}}{4} \sum_{p<y} \frac{\ln ^{2} p}{p}<\left(\frac{h}{2} \ln y\right)^{2}<\left(\frac{\varepsilon}{10 k}\right)^{2}, \\
& W_{1}<\frac{(2 k) !}{k !} M\left(\frac{\varepsilon}{10 \pi k}\right)^{2 k}+x<\frac{3}{2}\left(\frac{\varepsilon^{2}}{25 \pi^{2} e k}\right)^{k} M+x<\varepsilon^{2 k} M .
\end{aligned}
$$

The application of Hölder's inequality to the initial sum $W_{0}$ of the lemma yields:

$W_{0}=\sum_{N<n \leqslant N+M}\left(S\left(t_{n}+0\right)-S\left(t_{n-1}+0\right)\right)^{2 k} \leqslant W_{1}+k 2^{2 k-1}\left(W_{3}+W_{1}^{1-1 /(2 k)} W_{3}^{1 /(2 k)}\right)$,

where the sum

$$
W_{3}=\sum_{N<n \leqslant N+M}\left(R\left(t_{n}+0\right)-R\left(t_{n-1}+0\right)\right)^{2 k}
$$

was estimated in the proof of previous theorem. Using the above bounds of $W_{1}$ и $W_{3}$, we obtain:

$$
\begin{aligned}
& k 2^{2 k-1}\left(W_{3}+W_{1}^{1-1 /(2 k)} W_{3}^{1 /(2 k)}\right) \leqslant 1.5 M k(4 k \sqrt{B})^{2 k} \\
& W_{0} \leqslant \varepsilon^{2 k} M+1.5 M k(4 k \sqrt{B})^{2 k}<2 M k(4 k \sqrt{B})^{2 k} .
\end{aligned}
$$

The theorem is proved.

The above theorems imply the lower bound for the 'first moment' of the differences $S\left(t_{n}+0\right)-S\left(t_{n-1}+0\right)$.

THEOREM 3. There exists a positive constant $c_{1}=c_{1}(\varepsilon)$ such that

$$
\sum_{N<n \leqslant N+M}\left|S\left(t_{n}+0\right)-S\left(t_{n-1}+0\right)\right|>c_{1} M
$$

ProOF. Let us put for brevity $r(n)=S\left(t_{n}+0\right)-S\left(t_{n-1}+0\right)$ and denote by $V_{k}$ the sum of $k$ th powers of $|r(n)|$. Further, let $m=[\mu]+1$, where $\mu=\varepsilon^{-1} \exp \left(e^{76} \varepsilon^{-6}\right)$. Combining the identity

$$
S\left(t_{n+m}+0\right)-S\left(t_{n}+0\right)=r(n+1)+r(n+2)+\ldots+r(n+m)
$$


with Cauchy's inequality, we get

$$
\left(S\left(t_{n+m}+0\right)-S\left(t_{n}+0\right)\right)^{2} \leqslant m \sum_{\nu=1}^{m} r^{2}(n+\nu) .
$$

Summing both parts of the above relation over $n$, we have

$$
\begin{aligned}
\sum_{N<n \leqslant N+M}\left(S\left(t_{n+m}+0\right)-S\left(t_{n}+0\right)\right)^{2} & \leqslant m \sum_{\nu=1}^{m} \sum_{N<n \leqslant N+M} r^{2}(n+\nu) \leqslant \\
& \leqslant m \sum_{\nu=1}^{m} \sum_{N<n \leqslant N+M+m} r^{2}(n)=m^{2} \sum_{N<n \leqslant N+M+m} r^{2}(n) .
\end{aligned}
$$

Hence, by the Corollary of Theorem 1, we get:

$$
\sum_{N<n \leqslant N+M+m} r^{2}(n) \geqslant m^{-2} \sum_{N<n \leqslant N+M}\left(S\left(t_{n+m}+0\right)-S\left(t_{n}+0\right)\right)^{2} \geqslant 1.01 e^{73} \varepsilon^{-6} m^{-2} M .
$$

Since $|r(n)| \leqslant\left|S\left(t_{n}\right)\right|+\left|S\left(t_{n-1}\right)\right| \leqslant 18 \ln N$ for $N<n \leqslant N+M+m$ (see [23]), we have:

$$
V_{2}=\sum_{N<n \leqslant N+M} r^{2}(n) \geqslant 1.01 e^{73} \varepsilon^{-6} m^{-2} M-m(18 \ln N)^{2}>c_{2} M,
$$

where $c_{2}=e^{73} \varepsilon^{-6} m^{-2} M$. Further, the application of Hölder's inequality to the sum $V_{2}$ yields

$$
\begin{array}{r}
V_{2}=\sum_{N<n \leqslant N+M}|r(n)|^{2 / 3}|r(n)|^{4 / 3} \leqslant\left(\sum_{N<n \leqslant N+M}|r(n)|\right)^{2 / 3}\left(\sum_{N<n \leqslant N+M}|r(n)|^{4}\right)^{1 / 3}= \\
=V_{1}^{2 / 3} V_{4}^{1 / 3} .
\end{array}
$$

Therefore, $V_{1} \geqslant V_{2}^{3 / 2} V_{4}^{-1 / 2}$. Using both the above bound for $V_{2}$ and the inequality of Theorem 2 for $k=2$, we obtain:

$$
\begin{aligned}
& V_{4} \leqslant 2^{14} B^{2} M=c_{4} M \\
& V_{1}=\sum_{N<n \leqslant N+M}|r(n)| \geqslant \frac{\left(c_{2} M\right)^{3 / 2}}{\left(c_{4} M\right)^{1 / 2}}=c_{1} M, \quad c_{1}=c_{2}^{3 / 2} c_{4}^{-1 / 2} .
\end{aligned}
$$

Theorem is proved.

The following assertion is an analogue (for the short interval of summation) of corollary of Selberg's theorem cited in $\S 1$.

THEOREM 4. There exist positive constants $K_{1}$ and $K_{2}$ such that for $N<n \leqslant N+M$, there are more than $K_{1} M$ cases when the interval $G_{n}$ does not contain any ordinate of a zero of $\zeta(s)$, and more than $K_{2} M$ cases when the interval $G_{n}$ contains at least two ordinates, i.e.

$$
\nu_{0}(N+M)-\nu_{0}(N) \geqslant K_{1} M, \quad \sum_{k \geqslant 2}\left(\nu_{k}(N+M)-\nu_{k}(N)\right) \geqslant K_{2} M
$$


ProOf. Since $r(n)=S\left(t_{n}+0\right)-S\left(t_{n-1}+0\right)$ is an integer and $r(n) \geqslant-1$ for any $n$, then the equality (11) implies that the interval $G_{n}$ does not contain any ordinate iff $r(n)=-1$ and contains more than one ordinate iff $r(n) \geqslant 1$. In other words, the number $M_{1}$ of 'empty' Gram's intervals is equal to the number of $n$ such that $r(n)$ is negative, and the number $M_{2}$ of Gram's intervals that contain two or more ordinates is equal to the number of positive $r(n)$.

Using the relation

$$
\frac{1}{2}(|r(n)|-r(n))= \begin{cases}1, & \text { if } r(n)<0 \\ 0, & \text { if } r(n)>0\end{cases}
$$

by the bound of Theorem 3 we get

$$
\begin{aligned}
M_{1}= & \sum_{N<n \leqslant N+M} \frac{1}{2}(|r(n)|-r(n))=\frac{1}{2} \sum_{N<n \leqslant N+M}\left|S\left(t_{n}+0\right)-S\left(t_{n-1}+0\right)\right|- \\
& -\frac{1}{2} \sum_{N<n \leqslant N+M}\left(S\left(t_{n}+0\right)-S\left(t_{n-1}+0\right)\right) \geqslant \frac{c_{1}}{2} M-9 \ln N>K_{1} M,
\end{aligned}
$$

where $K_{1}=\frac{2}{5} c_{1}$. Further, $M_{2}$ is equal to the number of non-zero terms of the sum

$$
W=\sum_{N<n \leqslant N+M} \frac{1}{2}(|r(n)|+r(n)) .
$$

The application of Theorem 2 and Cauchy's inequality yields:

$$
W \leqslant \sqrt{M_{2}} \sqrt{\sum_{N<n \leqslant N+M} r^{2}(n)} \leqslant \sqrt{M_{2}} \sqrt{32 B M} .
$$

Since $W>K_{1} M$, then $M_{2} \geqslant K_{2} M$, where

$$
K_{2}=\frac{K_{1}^{2}}{32 B}
$$

Theorem is proved.

REMARK. The constants $K_{1}, K_{2}$ defined above are too small. It's easy to see that they do not exceed $\exp \left(-e^{75} \varepsilon^{-6}\right)$. At the same time, the calculations of zeros of $\zeta(s)$ shows that probably $K_{1}>0.1, K_{2}>0.1$. Thus, it is of some interest to prove the analogue of Theorem 4 with the constants $K_{1}$ and $K_{2}$ of order $0.001-0.01$.

\section{$\S 4$. The alternating sums connected with the function $S(t)$.}

Here we study the sums of the following type:

$$
T_{k}=\sum_{N<n \leqslant N+M} S^{k}\left(t_{n}+0\right)\left(S\left(t_{n}+0\right)-S\left(t_{n-1}+0\right)\right)
$$


Theorem 4 implies that the difference $r(n)=S\left(t_{n}+0\right)-S\left(t_{n-1}+0\right)$ is negative for positive proportion of $n, N<n \leqslant N+M$. At the same time, this difference is positive for positive proportion of $n$. Hence, the sums $T_{k}$ are alternating (at least, for even $k$ ).

The direct application of Cauchy's inequality does not allow us to take into account the oscillation in the sum $T_{k}$, and therefore does not allow us to obtain non-trivial bound for $T_{k}$. Thus, for example,

$$
\left|T_{2 k}\right| \leqslant\left(\sum_{N<n \leqslant N+M} S^{4 k}\left(t_{n}+0\right)\right)^{1 / 2}\left(\sum_{N<n \leqslant N+M} r^{2}(n)\right)^{1 / 2} \ll_{k} \sqrt{M L^{2 k}} \sqrt{M} \ll{ }_{k} M L^{k},
$$

and this bound is trivial. Hence, we need to use some additional arguments.

THEOREM 5. Suppose $k$ is an integer such that $1 \leqslant k \leqslant \sqrt{L}$. Then the following estimations hold:

$$
\left|T_{2 k-1}\right|<0.02(A k)^{k+1} M L^{k-1}, \quad\left|T_{2 k}\right|<0.02(10 A)^{k+1} \frac{(2 k) !}{k !} \frac{M L^{k-1 / 2}}{(2 \pi)^{2 k}} .
$$

Proof. Consider first the sum $T_{2 k-1}$. Setting $a=S\left(t_{n}+0\right), b=r(n)=S\left(t_{n}+0\right)-$ $S\left(t_{n-1}+0\right)$ in the easy-to-check identity

$$
(a-b)^{2 k}=a^{2 k}-2 k a^{2 k-1} b+\theta k^{2} 2^{2 k-2}\left(a^{2 k-2} b^{2}+b^{2 k}\right),
$$

after some obvious transformations we get:

$2 k S^{2 k-1}\left(t_{n}+0\right) r(n)=S^{2 k}\left(t_{n}+0\right)-S^{2 k}\left(t_{n-1}+0\right)+\theta k^{2} 2^{2 k-2}\left(r^{2 k}(n)+S^{2 k-2}\left(t_{n}+0\right) r^{2}(n)\right)$.

Summing over $n$, we obtain

$$
2 k T_{2 k-1}=S^{2 k}\left(t_{N+M}\right)-S^{2 k}\left(t_{N}\right)+\theta k^{2} 2^{2 k-2}\left(W_{1}+W_{2}\right),
$$

where

$$
W_{1}=\sum_{N<n \leqslant N+M} r^{2 k}(n), \quad W_{2}=\sum_{N<n \leqslant N+M} S^{2 k-2}\left(t_{n}+0\right) r^{2}(n) .
$$

By theorem 2,

$$
W_{1} \leqslant 2 M k(4 k \sqrt{B})^{2 k}
$$

Using both the estimation of Lemma 5 and Hölder's inequality, we have:

$$
W_{2} \leqslant\left(\sum_{N<n \leqslant N+M} S^{2 k}\left(t_{n}+0\right)\right)^{1-1 / k} W_{1}^{1 / k} \leqslant 2 \sqrt[3]{3}(4 k \sqrt{B})^{2}\left(\frac{k A L}{\pi^{2} e}\right)^{k-1} M .
$$

Hence

$$
\begin{aligned}
& k 2^{2 k-2}\left(W_{1}+W_{2}\right) \leqslant k 2^{2 k-2} 2 \sqrt[3]{3}(4 k \sqrt{B})^{2}\left(\frac{k A L}{\pi^{2} e}\right)^{k-1} M\left(1+\frac{k}{\sqrt[3]{3}}\left(\frac{k A}{4 L}\right)^{k-1}\right)< \\
& <2 \cdot 4^{2} \sqrt[3]{3} e^{-8} k^{3} A^{2}\left(\frac{4 k A L}{\pi^{2} e}\right)^{k-1}<\frac{1}{30} k^{3} A^{2}(0.15 k A L)^{k-1} M \leqslant \frac{1}{30}(A k)^{k+1} M L^{k-1}
\end{aligned}
$$


and therefore

$$
\left|T_{2 k-1}\right| \leqslant \frac{1}{60}(A k)^{k+1} M L^{k-1}+\frac{1}{k}(9 \ln N)^{2 k}<\frac{1}{50}(A k)^{k+1} M L^{k-1} .
$$

Now we consider the sum $T_{2 k}$. Setting $a=S\left(t_{n}+0\right), b=r(n)$ in the identity

$$
(a-b)^{2 k+1}=a^{2 k+1}-(2 k+1) a^{2 k} b+\theta k(2 k+1) 2^{2 k-2}\left(|b|^{2 k+1}+|a|^{2 k-1} b^{2}\right),
$$

after some transformations we get:

$$
(2 k+1) T_{2 k+1}=S^{2 k+1}\left(t_{N+M}\right)-S^{2 k+1}\left(t_{N}\right)+\theta k(2 k+1) 2^{2 k-2}\left(W_{1}+W_{2}\right),
$$

where

$$
W_{1}=\sum_{N<n \leqslant N+M}|r(n)|^{2 k+1}, \quad W_{2}=\sum_{N<n \leqslant N+M}\left|S\left(t_{n}+0\right)\right|^{2 k-1} r^{2}(n) .
$$

We have

$$
\begin{aligned}
& W_{1}=\sum_{N<n \leqslant N+M}|r(n)|^{2 k-1} r^{2}(n) \leqslant\left(\sum_{N<n \leqslant N+M} r^{2 k}(n)\right)^{1-1 /(2 k)}\left(\sum_{N<n \leqslant N+M} r^{4 k}(n)\right)^{1 /(2 k)} \\
& \leqslant\left(2 k(4 k \sqrt{B})^{2 k}\right)^{1-1 /(2 k)}\left(4 k(8 k \sqrt{B})^{4 k}\right)^{1 /(2 k)} M<8 \sqrt{2} k M(4 k \sqrt{B})^{2 k+1}= \\
& =\frac{(2 k) !}{k !} \frac{M}{(2 \pi)^{2 k}}(A L)^{k-1 / 2} \delta_{1},
\end{aligned}
$$

where

$$
\delta_{1}=\frac{(2 \pi)^{2 k} k !}{(2 k) !} \frac{8 \sqrt{2} k(4 k \sqrt{B})^{2 k+1}}{(A L)^{k-1 / 2}} \leqslant k^{2} A \sqrt{A L}\left(\frac{(4 \pi)^{2} A k}{e^{7} L}\right)^{k}<1 .
$$

Next, the application of Lemmas 4 and 5 yields:

$$
\begin{aligned}
& W_{2} \leqslant\left(\sum_{N<n \leqslant N+M} S^{2 k}\left(t_{n}+0\right)\right)^{1-1 /(2 k)}\left(\sum_{N<n \leqslant N+M} r^{4 k}(n)\right)^{1 /(2 k)} \leqslant \\
& \leqslant\left(\frac{1.1}{A} \frac{(2 k) !}{(2 \pi)^{2 k} k !}(A L)^{k}\right)^{1-1 /(2 k)}(4 k)^{1 /(2 k)}(8 k \sqrt{B})^{2} \leqslant \frac{(2 k) !}{k !} \frac{M}{(2 \pi)^{2 k}}(A L)^{k-1 / 2} \delta_{2},
\end{aligned}
$$

where

$$
\delta_{2}=2 \pi\left(\frac{k !}{(2 k) !}\right)^{1 /(2 k)} \sqrt{\frac{1.1}{A}}(4 k)^{1 /(2 k)}(8 k \sqrt{B})^{2}<0.01(k A)^{3 / 2} .
$$

Let us note that

$$
\begin{aligned}
& k 2^{2 k-2}\left(W_{1}+W_{2}\right) \leqslant k 2^{2 k-2} \frac{(2 k) !}{k !} \frac{M}{(2 \pi)^{2 k}}(A L)^{k-1 / 2}\left(\delta_{1}+\delta_{2}\right)< \\
& <\frac{(2 k) !}{k !} \frac{M L^{k-1 / 2}}{(2 \pi)^{2 k}} \cdot 0.01(10 A)^{k+1} .
\end{aligned}
$$


Therefore,

$$
\begin{aligned}
& (2 k+1)\left|T_{2 k}\right| \leqslant(2 k+1) \cdot 0.01(10 A)^{k+1} \frac{(2 k) !}{k !} \frac{M L^{k-1 / 2}}{(2 \pi)^{2 k}}+2(9 \ln N)^{2 k+1} \\
& \left|T_{2 k}\right|<0.02(10 A)^{k+1} \frac{(2 k) !}{k !} \frac{M L^{k-1 / 2}}{(2 \pi)^{2 k}}
\end{aligned}
$$

Theorem is proved.

The following lemma is necessary for the proof of Selberg's formulas.

LEMMA 9. Let $k$ and $n$ be an arbitrary natural numbers and suppose that the interval $G_{n}=\left(t_{n-1}, t_{n}\right]$ contains $(r+1)$ ordinates of zeros of $\zeta(s), r=r(n) \geqslant-1$. Then the following relations hold:

$$
\begin{gathered}
\sum_{t_{n-1}<\gamma_{m} \leqslant t_{n}} \Delta_{m}^{2 k}=(r+1) \Delta^{2 k}(n)+\theta_{1} k 2^{2 k}\left(|\Delta(n)|^{2 k-1} r^{2}+|r|^{2 k+1}\right), \\
\sum_{t_{n-1}<\gamma_{m} \leqslant t_{n}} \Delta_{m}^{2 k-1}=-(r+1) \Delta^{2 k-1}(n)+\theta_{2} k 2^{2 k}\left(\Delta^{2 k-2}(n) r^{2}+r^{2 k}\right) .
\end{gathered}
$$

Proof. First we consider the case $r=-1$. Then $G_{n}$ does not contain any ordinate, and the sums in the left-hand sides of $(13),(14)$ are empty. Thus the assertion of lemma is true for $\theta_{1}=\theta_{2}=0$.

Now let us consider the case $r \geqslant 0$. Suppose that the inequalities

$$
\gamma_{s-1} \leqslant t_{n-1}<\gamma_{s} \leqslant \gamma_{s+1} \leqslant \ldots \leqslant \gamma_{s+r} \leqslant t_{n}<\gamma_{s+r+1}
$$

hold for some $s \geqslant 1$. Then $\Delta(n)=S\left(t_{n}+0\right)=N\left(t_{n}+0\right)-\pi^{-1} \vartheta\left(t_{n}\right)-1=s+r-n$, and hence

$$
\begin{aligned}
& \Delta_{s}=n-s=r-\Delta(n), \\
& \Delta_{s+1}=n-s-1=r-1-\Delta(n), \\
& \cdots \\
& \Delta_{s+r}=n-s-r=-\Delta(n) .
\end{aligned}
$$

Therefore,

$$
\begin{aligned}
& \sum_{t_{n-1}<\gamma_{m} \leqslant t_{n}} \Delta_{m}^{2 k}=\sum_{m=s}^{s+r} \Delta_{m}^{2 k}= \\
&=\sum_{j=0}^{r}(j-\Delta(n))^{2 k}=\sum_{j=0}^{r}\left(\Delta^{2 k}(n)+\theta 2 k j 2^{2 k-2}\left(|\Delta(n)|^{2 k-1}+j^{2 k-1}\right)\right)= \\
&=(r+1) \Delta^{2 k}(n)+\theta_{1} k 2^{2 k}\left(|\Delta(n)|^{2 k-1} r^{2}+r^{2 k+1}\right) .
\end{aligned}
$$

The proof of (14) follows the same arguments. Lemma is proved. 
The below theorem reduces the calculation of the sums (8),(9) to the calculation of the sums of the quantities $\Delta^{k}(n)=S^{k}\left(t_{n}+0\right)$ (see Lemma 5).

THEOREM 6. Let $k$ be an integer such that $1 \leqslant k \leqslant \sqrt{L}$. Then the following relations hold:

$$
\begin{aligned}
& \sum_{N<n \leqslant N+M} \Delta_{n}^{2 k}=\frac{(2 k) !}{k !} \frac{M L^{k}}{(2 \pi)^{2 k}}\left(1+\theta(10 A)^{k+1} L^{-0.5}\right), \\
& \left|\sum_{N<n \leqslant N+M} \Delta_{n}^{2 k-1}\right| \leqslant e^{9}(B k)^{k} M L^{k-1} .
\end{aligned}
$$

Proof. The numbers $\mu, \nu$ are uniquely defined by the conditions

$$
\gamma_{\mu} \leqslant t_{N}<\gamma_{\mu+1}, \quad \gamma_{\nu} \leqslant t_{N+M}<\gamma_{\nu+1} .
$$

Let us consider the sum

$$
V=\sum_{\mu<m \leqslant \nu} \Delta_{m}^{2 k} .
$$

Using the definition of $\Delta(n)$, we obtain:

$$
\begin{aligned}
& \mu=N\left(t_{N}+0\right)=\pi^{-1} \vartheta\left(t_{N}\right)+1+S\left(t_{N}+0\right)=N+\Delta(N), \\
& |\mu-N|=|\Delta(N)|<9 \ln N,
\end{aligned}
$$

and, similarly, $|\nu-(N+M)|=|\Delta(N+M)|<9 \ln N$. These inequalities and the bound $\left|\Delta_{m}\right|<9 \ln N$ (see Lemma 7 and a posterior remark in [18]) imply that the difference between the sum $V$ and the sum

$$
\sum_{N<m \leqslant N+M} \Delta_{m}^{2 k}
$$

does not exceed in modulus

$$
(18 \ln N+1)(9 \ln N)^{2 k}<3(9 \ln N)^{2 k+1} .
$$

On the other hand, Lemma 9 implies that

$$
\begin{aligned}
V & =\sum_{N<n \leqslant N+M} \sum_{t_{n-1}<\gamma_{m} \leqslant t_{n}} \Delta_{m}^{2 k}= \\
& =\sum_{N<n \leqslant N+M}\left((r(n)+1) \Delta^{2 k}(n)+\theta k 2^{2 k}\left(|\Delta(n)|^{2 k-1} r^{2}(n)+|r(n)|^{2 k+1}\right)\right)= \\
& =\sum_{N<n \leqslant N+M} \Delta^{2 k}(n)+T_{2 k}+\theta_{1} k 2^{2 k}\left(W_{1}+W_{2}\right),
\end{aligned}
$$

where $r(n)=S\left(t_{n}+0\right)-S\left(t_{n-1}+0\right)$ and

$$
\begin{aligned}
W_{1} & =\sum_{N<n \leqslant N+M}|r(n)|^{2 k+1}=\sum_{N<n \leqslant N+M}\left|S\left(t_{n}+0\right)-S\left(t_{n-1}+0\right)\right|^{2 k+1}, \\
W_{2} & =\sum_{N<n \leqslant N+M}|\Delta(n)|^{2 k-1} r^{2}(n)=\sum_{N<n \leqslant N+M}\left|S\left(t_{n}+0\right)\right|^{2 k-1}\left(S\left(t_{n}+0\right)-S\left(t_{n-1}+0\right)\right)^{2} .
\end{aligned}
$$


Proving Theorem 5, we found that

$$
\begin{aligned}
& \left|T_{2 k}\right| \leqslant 0.02(10 A)^{k+1} \frac{(2 k) !}{k !} \frac{M L^{k-0.5}}{(2 \pi)^{2 k}}, \\
& k 2^{2 k}\left(W_{1}+W_{2}\right)<0.08(10 A)^{k+1} \frac{(2 k) !}{k !} \frac{M L^{k-0.5}}{(2 \pi)^{2 k}} .
\end{aligned}
$$

Using both these inequalities and the assertion of Lemma 5 , we get:

$$
\begin{aligned}
\sum_{N<m \leqslant N+M} \Delta_{m}^{2 k} & =\sum_{N<n \leqslant N+M} \Delta^{2 k}(n)+0.1 \theta_{1}(10 A)^{k+1} \frac{(2 k) !}{k !} \frac{M L^{k-0.5}}{(2 \pi)^{2 k}}+3 \theta_{2}(9 \ln N)^{2 k+1}= \\
= & \frac{(2 k) !}{k !} \frac{M L^{k}}{(2 \pi)^{2 k}}\left(1+\theta_{3} A^{k} L^{-0.5}+0.2 \theta_{4}(10 A)^{k+1} L^{-0.5}\right)= \\
& =\frac{(2 k) !}{k !} \frac{M L^{k}}{(2 \pi)^{2 k}}\left(1+\theta(10 A)^{k+1} L^{-0.5}\right) .
\end{aligned}
$$

Applying the same arguments, we obtain

$$
V=\sum_{\mu<m \leqslant \nu} \Delta_{m}^{2 k-1}=-\sum_{N<n \leqslant N+M} \Delta^{2 k-1}(n)-T_{2 k-1}+\theta k 2^{2 k}\left(V_{1}+V_{2}\right),
$$

where

$$
V_{1}=\sum_{N<n \leqslant N+M} r^{2 k}(n), \quad \sum_{N<n \leqslant N+M} \Delta^{2 k-2}(n) r^{2}(n) .
$$

Proving Theorem 5, we also found that

$$
k 2^{2 k}\left(V_{1}+V_{2}\right) \leqslant 0.14(A k)^{k+1} M L^{k-1}, \quad\left|T_{2 k-1}\right|<0.02(A k)^{k+1} M L^{k-1} .
$$

By these estimations and by the inequalities of Lemma 5 we have:

$$
\begin{gathered}
\left|\sum_{N<m \leqslant N+M} \Delta_{m}^{2 k-1}\right| \leqslant\left|\sum_{N<n \leqslant N+M} \Delta^{2 k-1}(n)\right|+\left|T_{2 k-1}\right|+k 2^{2 k}\left(V_{1}+V_{2}\right)+3(9 \ln N)^{2 k}, \leqslant \\
\leqslant \frac{3.5}{\sqrt{B}}(B k)^{k} M L^{k-1}+0.16(A k)^{k+1} M L^{k-1}= \\
=(B k)^{k} M L^{k-1}\left(\frac{3.5}{\sqrt{B}}+0.16 A k\left(\frac{e^{8}}{A}\right)^{k}\right)<e^{9}(B k)^{k} M L^{k-1} .
\end{gathered}
$$

Theorem is proved.

The approximate expression for the distribution function of discrete random quantity with the values

$$
\delta_{n}=\pi \Delta_{n} \sqrt{\frac{2}{L}}, \quad N<n \leqslant N+M,
$$

and the proof of the assertion that $\Delta_{n} \neq 0$ for 'almost all' follow from Theorem 6 by standard technic (see, for example, Theorem 4 from [17]). 


\section{$\S 5$. On some equivalents of 'almost Riemann hypothesis'}

The last section is devoted to some new equivalents of 'almost Riemann hypothesis'. This hypothesis asserts that 'almost all' complex zeros of $\zeta(s)$ are on the critical line, that is

$$
\lim _{T \rightarrow+\infty} \frac{N_{0}(T)}{N(T)}=1 .
$$

Moreover, the below arguments imply that Selberg interpreted Gram's law in [8] in a way different from Titchmarsh's one. Namely, the below assertions show that Selberg considered all the complex zeros of $\zeta(s)$ (but not only the zeros on the critical line) in dealing with the quantities $\Delta_{n}$. Thus, the Selberg's definition of $\Delta_{n}$ is equivalent to the Definition 4.

Suppose that $0<c_{1}<c_{2}<\ldots \leqslant c_{n} \leqslant c_{n+1} \leqslant \ldots$ are the ordinates of zeros of $\zeta(s)$, lying on the critical line and counting with theirs multiplicities. For a fixed $n \geqslant 1$, we define the number $m=m(n)$ by the inequalities

$$
t_{m-1}<c_{n} \leqslant t_{m}
$$

and set $D_{n}=m-n$. Of course, if Riemann hypothesis is true then $c_{n}=\gamma_{n}$ and $D_{n}=\Delta_{n}$ for any $n$.

THEOREM 7. The validity of the relation

$$
\sum_{n \leqslant N}\left|D_{n}\right|=o\left(N^{2}\right)
$$

as $N \rightarrow+\infty$, is the necessary and sufficient condition for the truth of 'almost Riemann hypothesis'.

Proof. Suppose that 'almost Riemann hypothesis' is true. Then

$$
N\left(c_{n}+0\right)=(1+o(1)) N_{0}\left(c_{n}+0\right)=(1+o(1))(n+O(\ln n))=n+o(n)
$$

(by the term $O(\ln n)$, we take into account the possible multiplicity of the zero with the ordinate $c_{n}$; by Lemma 8 , this multiplicity does not exceed in order $\left.\ln c_{n}=O(\ln n)\right)$. On the other hand, by (15) we have:

$$
m-1+S\left(t_{m-1}+0\right)<N\left(c_{n}+0\right) \leqslant m+S\left(t_{m}+0\right),
$$

and hence $D_{n}=m-n=o(n)+O(\ln m)=o(n)$. Therefore,

$$
\sum_{n \leqslant N}\left|D_{n}\right|=o\left(N^{2}\right)
$$

Suppose now that the condition (16) is satisfied. Noting that $N\left(c_{n}+0\right) \geqslant n+\varepsilon(n)$, where $\varepsilon(n)$ is the number of zeros of $\zeta(s)$ with the condition $0<\operatorname{Im} s \leqslant c_{n}$, $\operatorname{Re} s \neq \frac{1}{2}$, we get:

$$
n+\varepsilon(n) \leqslant N\left(t_{m}+0\right)=m+S\left(t_{m}+0\right) .
$$


Hence, $0 \leqslant \varepsilon(n) \leqslant\left|D_{n}\right|+\left|S\left(t_{m}+0\right)\right|$. Summing this estimation over $n \leqslant 2 N$ and applying Cauchy's inequality, we obtain:

$$
\sum_{n \leqslant 2 N} \varepsilon(n) \leqslant \sum_{n \leqslant 2 N}\left|D_{n}\right|+\sum_{\substack{n \leqslant 2 N \\ m=m(n)}}\left|S\left(t_{m}+0\right)\right| \leqslant \sqrt{2 N} \sqrt{W}+o\left(N^{2}\right),
$$

where

$$
W=\sum_{\substack{n \leqslant 2 N \\ m=m(n)}} S^{2}\left(t_{m}+0\right)
$$

Let $\mu$ be the maximum value of $m(n)$ for $n \leqslant 2 N$. Then $t_{\mu-1}<c_{2 N} \leqslant t_{\mu}$ and hence

$$
N\left(c_{2 N}\right) \geqslant N\left(t_{\mu-1}\right)=\mu+O(\ln \mu) .
$$

By Lemma 8 , we have for $t=c_{2 N}$ :

$$
N\left(c_{2 N}\right) \leqslant\left(\frac{5}{2}-10^{-3}\right) N_{0}\left(c_{2 N}\right)=\left(\frac{5}{2}-10^{-3}\right)(2 N+O(\ln N)),
$$

and therefore $\mu<5 N$. Changing the order of summation in $W$, we obtain:

$$
W \leqslant \sum_{l \leqslant 5 N} S^{2}\left(t_{l}+0\right) \sum_{\substack{n \leqslant 2 N \\ m(n)=l}} 1
$$

For a fixed $l$, the number of $n$ that satisfy the conditions $n \leqslant 2 N, m(n)=l$, does not exceed the number of all ordinates of zeros of $\zeta(s)$ in the interval $\left(t_{l-1}, t_{l}\right]$, that is

$$
N\left(t_{l}+0\right)-N\left(t_{l-1}+0\right)=1+S\left(t_{l}+0\right)-S\left(t_{l-1}+0\right)=1+r(l) .
$$

Thus we have:

$$
W \leqslant \sum_{l \leqslant 5 N} S^{2}\left(t_{l}+0\right)(1+r(l)) .
$$

Using both the first formula of Lemma 5 and the estimation of Theorem 5 , we find that

$$
\begin{gathered}
W \leqslant \frac{5 N}{2 \pi^{2}} \ln \ln N+O(N \sqrt{\ln \ln N})<\frac{1}{3} N \ln \ln N, \\
\sum_{n \leqslant 2 N} \varepsilon(n)<N \sqrt{\ln \ln N}+o\left(N^{2}\right)=o\left(N^{2}\right) .
\end{gathered}
$$

By obvious inequality $\varepsilon(N+1)+\varepsilon(N+2)+\ldots+\varepsilon(2 N) \geqslant N \varepsilon(N)$ we get:

$$
N \varepsilon(N)=o\left(N^{2}\right), \quad \varepsilon(N)=o(N) .
$$

Suppose now $t$ is a sufficiently large. Then, defining $N$ from the inequalities $c_{N-1}<t \leqslant c_{N}$ and using the above relations, we obtain:

$$
N(t) \geqslant N-1, \quad N(t)-N_{0}(t) \leqslant \varepsilon(N)=o(N)=o(N(t)) .
$$


The theorem is proved.

COOLLARY 1. The validity of the relation

$$
\sum_{n \leqslant N}\left|D_{n}\right|^{k}=o\left(N^{k+1}\right), \quad N \rightarrow+\infty
$$

for at least one fixed value of $k \geqslant 1$ is the necessary and sufficient condition for the truth of the 'almost Riemann hypothesis'

The proof is similar to the previous one. The difference is that we should use the inequality

$$
N \varepsilon(N) \leqslant \sum_{n \leqslant 2 N}\left|D_{n}\right|+N \sqrt{\ln \ln N} \leqslant(2 N)^{1-1 / k}\left(\sum_{n \leqslant 2 N}\left|D_{n}\right|^{k}\right)^{1 / k}+N \sqrt{\ln \ln N}
$$

for the proof of sufficiency.

This assertion shows, in particular, that if Selberg's formulas (8),(9) hold true after the replacement of the quantities $\Delta_{n}$ by $D_{n}$, then the 'almost Riemann hypothesis' is also true.

COROllary 2. The assertion ' $D_{n}=o(n)$ as $n \rightarrow+\infty$ ' is the necessary and sufficient condition for the 'almost Riemann hypothesis'.

The below theorem shows that the upper bound for $D_{n}$ causes the main difficulty.

THEOREM 8. Suppose that $N_{0}(t)>\varkappa N(t)$ for any $t>t_{0}>1$ and for some constant $\varkappa, 0<\varkappa<1$. Then the following inequalities hold for all sufficiently large $n$ :

$$
-9 \ln n \leqslant D_{n} \leqslant\left(\frac{1}{\varkappa}-1\right) n+9\left(\frac{1}{\varkappa}+1\right) \ln n .
$$

ProOF. By (15), we get:

$$
N\left(c_{n}+0\right) \geqslant N\left(t_{m-1}+0\right)=m-1+S\left(t_{m-1}+0\right) .
$$

By the assumption of theorem, we get for $t=c_{n}+0$ :

$$
N\left(c_{n}+0\right)<\frac{1}{\varkappa} N_{0}\left(c_{n}+0\right) \leqslant \frac{1}{\varkappa}\left(n+\kappa_{n}\right),
$$

where $\kappa_{n}$ denotes the multiplicity of the ordinate $c_{n}$. Comparing (17) and (18) and using the inequality $|S(t)| \leqslant 8.9 \ln t$, we obtain:

$$
m \leqslant \frac{1}{\varkappa} n+9\left(\frac{1}{\varkappa}+1\right) \ln n, \quad D_{n}=m-n<\left(\frac{1}{\varkappa}-1\right) n+9\left(\frac{1}{\varkappa}+1\right) .
$$

On the other hand,

$$
n \leqslant N_{0}\left(c_{n}+0\right) \leqslant N\left(c_{n}+0\right) \leqslant N\left(t_{m}+0\right)=m+S\left(t_{m}+0\right),
$$


and therefore $D_{n} \geqslant-S\left(t_{m}+0\right) \geqslant-9 \ln n$. Theorem is proved.

In [8], Selberg referred to the formulas

$$
\liminf _{n \rightarrow+\infty} \Delta_{n}=-\infty, \quad \limsup _{n \rightarrow+\infty} \Delta_{n}=+\infty
$$

as Tithmarsh's result from [5]. Indeed, the relations (19) hold true, and the modern omega-theorems for the function $S(t)$ imply a much deeper result, namely

$$
\Delta_{n}=\Omega_{ \pm}\left(\sqrt[3]{\frac{\ln n}{\ln \ln n}}\right)
$$

as $n$ growths (see [18]). On the other hand, in [5], Titchmarsh considered the fractions

$$
\tau_{n}=\frac{c_{n}-t_{n}}{t_{n+1}-t_{n}}
$$

instead of the quantities $\Delta_{n}$ (one can easily see that the difference between $\tau_{n}$ and $D_{n}$ is $O(1)$ ), and established the unboundedness of $\tau_{n}$. As far as can be seen, the methods of [5] allows one to show only that $\tau_{n} \neq O(1)$ and $D_{n} \neq O(1)$, as $n \rightarrow+\infty$. Slight modification of these methods and the omega-theorems for $S(t)$ lead to the following assertion

$$
D_{n}=\Omega_{-}\left(\sqrt[3]{\frac{\ln n}{\ln \ln n}}\right), \quad n \rightarrow+\infty .
$$

So, the problem of unboundedness of $D_{n}$ from above remains still open.

\section{Bibliography}

[1] J.-P. Gram, "Sur les Zéros de la Fonction $\zeta(s)$ de Riemann", Acta Math., 27(1903), 289-304.

[2] C. L. Siegel, "Über Riemanns Nachlaß zur analytischen Zahlentheorie", Quellen und Studien zur Geshichte der Mathematik, Astronomie und Physik, B. 2(1932), 45-80.

[3] J.-P. Gram, "Note sur le calcul de la fonction $\zeta(s)$ de Riemann", Bulletin de l'Académie de Copenhague, 1895, p. 303.

[4] J. I. Hutchinson, "On the roots of the Riemann zeta-function", Trans. Amer. Math. Soc., 27(1925), 49-60.

[5] E. C. Titchmarsh, "The zeros of the Riemann zeta-function", Proc. Roy. Soc. London Ser. A, 151(1935), 234-255.

[6] E. C. Titchmarsh, "The zeros of the Riemann zeta-function", Proc. Roy. Soc. London Ser. A, 157(1936), 261-263. 
[7] J. Kalpokas, J. Steuding, "On the value distribution of the Riemann zeta-function on the critical line", Moscow Journal of Combinatorics and Number Theory, 1(2011), № $1,26-42$.

[8] A. Selberg, "The zeta-function and the Riemann hypothesis", C.R. Dixième Congrès Math. Skandinaves (1946), 10, Jul. Gjellerups Forlag, Copenhagen, 1947, 187-200.

[9] E. C. Titchmarsh, "On van der Corput's method and the zeta-function of the Riemann (IV)", Quart. J. Math., 5(1934), 98-105.

[10] H. Bohr, E. Landau, "Beiträge zur Theorie der Riemannschen Zetafunktion", Math. Ann., 74(1913), 3-30.

[11] A. Selberg, "Contributions to the theory of the Riemann zeta-function", Archiv Math. Naturvid., 48:5(1946), 89-155.

[12] A. Selberg, "On the remainder in the formula for $N(T)$, the number of zeros of $\zeta(s)$ in the strip $0<t<T$ ", Avhandlinger utgitt av Det Norske Videnskaps - Akademi $i$ Oslo. Matematisk - Naturvidenskapelig Klasse, 1944, No. 1, 1-27.

[13] H. von Mangoldt, "Zur Verteilung der Nullstellen der Riemannschen Funktion $\xi(t)$ ", Math. Ann., 60(1905), 1-19.

[14] T. S. Trudgian, "Further results on Gram's law", DPhil Thesis, University of Oxford, 2009.

[15] A. Fujii, "Gram's law for the zeta zeros and the eigenvalues of Gaussian unitary ensembles", Proc. Japan Acad. Ser. A, 63(1987), 392-395.

[16] A. Selberg, Collected papers. Vol. I, Springer-Verlag, Berlin Heidelberg, 1989.

[17] M. A. Korolev, "The Gram law and Selberg's conjecture on the distribution of zeros of the Riemann zeta-function", Izv. Math. 74:4 (2010).

[18] M. A. Korolev, "On the Gram's Law in the Theory of Riemann Zeta Function", arXiv:1011.3997v1, to appear in: Izv. Math. .

[19] J. B. Rosser, L. Schoenfeld, "Approximate formulas for some functions of prime numbers", Illinois J. Math., 6:1 (1962), 64-94.

[20] A. Ghosh, "On Riemann's zeta-function - sign-changes of $S(T)$ ", Recent progress in analytic number theory (Durham, 1979), 1, Academic Press, London, New York, 1981, $25-46$.

[21] A. A. Karatsuba, M. A. Korolev, "Behaviour of the argument of the Riemann zeta function on the critical line", Russian Math. Surveys, 61:3 (2006), 389-482.

[22] J. B. Conrey, "More than two fifth of the zeros of the Riemann zeta-function are on the critical line", J. reine angew. Math., 399(1989), 1-26. 
[23] A. A. Karatsuba, M. A. Korolev, "The argument of the Riemann zeta function", Russian Math. Surveys, 60:3 (2005), 433-488. 